\title{
Ageostrophic instability in a rotating stratified interior jet
}

\author{
Claire Ménesguen $\dagger$, J. C. McWilliams and M. J. Molemaker
}

IGPP, UCLA, 405 Hilgard Avenue, Los Angeles, CA 90095-1567, USA

(Received 27 July 2011; revised 15 June 2012; accepted 2 August 2012;

first published online 28 September 2012)

Oceanic large- and meso-scale flows are nearly balanced in forces between Earth's rotation and density stratification effects (i.e. geostrophic, hydrostatic balance associated with small Rossby and Froude numbers). In this regime advective crossscale interactions mostly drive energy toward larger scales (i.e. inverse cascade). However, viscous energy dissipation occurs at small scales. So how does the energy reservoir at larger scales leak toward small-scale dissipation to arrive at climate equilibrium? Here we solve the linear instability problem of a balanced flow in a rotating and continuously stratified fluid far away from any boundaries (i.e. an interior jet). The basic flow is unstable not only to geostrophic baroclinic and barotropic instabilities, but also to ageostrophic instabilities, leading to the growth of small-scale motions that we hypothesize are less constrained by geostrophic cascade behaviours in a nonlinear regime and thus could escape from the inverse energy cascade. This instability is investigated in the parameter regime of moderate Rossby and Froude numbers, below the well-known regimes of gravitational, centrifugal, and Kelvin-Helmholtz instability. The ageostrophic instability modes arise with increasing Rossby number through a near-degeneracy of two unstable modes with coincident phase speeds. The near-degeneracy occurs in the neighbourhood of an identified criterion for the non-integrability of the 'isentropic balance equations' (namely $A-S=0$ with $A$ the absolute vertical vorticity and $S$ the horizontal strain rate associated with the basic flow), beyond which development of an unbalanced component of the flow is expected. These modes extract energy from the basic state with large vertical Reynolds stress work (unlike geostrophic instabilities) and act locally to modify the basic flow by reducing the isopycnal Ertel potential vorticity gradient near both its zero surface and its critical surface (phase speed equal to basic flow speed).

Key words: baroclinic flows, critical layers, stratified flows

\section{Introduction}

The oceanic general circulation and its meso-scale eddies are forced mainly at planetary scales where rotation and stratification have strong influences. This regime is often referred to as balanced dynamics because of the importance of the geostrophic, hydrostatic force balance, which is known to inhibit energy transfer towards smaller scales (Charney 1971). Dissipation is necessary for climate equilibration with the 
forcing. However, viscous energy dissipation can only occur at very small scales where the loss of rotation and stratification influences leads to three-dimensional isotropic motion in Kolmogorov's paradigm for turbulence. McWilliams (2008) reviews several possible routes for transition between the large- and small-scale dynamical regimes. One of them, called the 'interior route to dissipation', is a forward cascade of energy toward small scales. It implies an escape from rotational control to non-rotating, unbalanced dynamics.

The concept of balance has quasi-geostrophic dynamics as its asymptotic regime for strong rotation and stratification, but it has useful generalizations to higher-order approximation models, called balance equations. Balance equations are characterized by solvability (invertibility, integrability) conditions that are violated when rotation and stratification effects are sufficiently weak, as expected at small scales. The hypothesis of the interior route to dissipation is that energy transfers occur across these threshold conditions, beyond which the dynamics will be wholly or partially unbalanced.

A mathematical framework for this transition is proposed in McWilliams et al. (1998) as a breakdown in consistent balanced evolution in rotating, stratified fluids away from boundaries. The limiting conditions for the time-integrability of the conservative isentropic balance equations are sign changes within the spatial domain of any of three quantities: (i) the density stratification $N^{2}$ (i.e. the gravitational stability threshold); (ii) Ertel potential vorticity $P V$ (i.e. the centrifugal instability threshold); or (iii) $A-S$, where $A$ is the absolute vertical vorticity and $S>0$ is the magnitude of the horizontal strain rate of the horizontal flow. This third criterion has also been identified by Dritschel \& Viúdez (2003) for an alternative set of balance equations, where the equivalent condition is the change of sign of the so-called Rellich parameter. None of these conditions is violated for strong rotation and stratification. Gravitational instability initiates convection, which is often induced by destabilizing surface buoyancy fluxes. Centrifugal instability (ii) is favoured in regions with small potential vorticity, e.g. fronts in the surface boundary layer (Capet et al. 2008; Taylor \& Ferrari 2009; Thomas \& Taylor 2010) or near the equator (Hua, Moore \& Le Gentil 1997; Ménesguen et al. 2009). The stability consequences of violating criterion (iii) are less well understood. In some examples, (iii) has been shown to be approximately associated (rather than having a sharp onset threshold) with what may be collectively called anticyclonic ageostrophic instability, because $A$ is smallest where the flow is anticyclonic: a barotropic elliptical flow in an unbounded domain (McWilliams \& Yavneh 1998), barotropic boundary currents (Molemaker, McWilliams \& Yavneh 2001; Yavneh, McWilliams \& Jeroen Molemaker 2001; McWilliams, Molemaker \& Yavneh 2004), and the baroclinic Eady flow (Molemaker, McWilliams \& Yavneh 2005).

This paper presents examples of linear destabilization of a geostrophic basic flow into ageostrophic fluctuations in the neighbourhood of a threshold condition where the evolution must be expected to manifest some unbalance, thus crossing the border out of the realm of rotational dominance. Geostrophic instability modes are also found, but they do not have the implication of loss of balance and forward energy cascade. Because this and other instability calculations are linear, they are not enough to assess whether the subsequent nonlinear turbulent evolution would manifest a forward energy cascade.

Many other examples have been adduced of ageostrophic rotating, stratified, shear instability. Well-known types are gravitational, centrifugal, and Kelvin-Helmholtz instabilities, all of which are unbalanced in the sense defined above. Here we take the view that these instabilities may not be the first ones encountered as rotational and stratification influences weaken, hence our focus is on the regime before their onset 
conditions. Because instability problems for a continuous shear profile are difficult, especially when the basic flow is multi-dimensional (non-separable), most published examples are for simplified basic flows, such as a barotropic current, uniform $P V$ (e.g. as in Eady's flow), or discretized into one or two uniform-density layers in the vertical direction. Furthermore, highly idealized boundaries often play a dominant role, either horizontal sides or the equator (which support Kelvin waves), layer interfacial 'outcrops' of zero thickness (which support frontal waves), or vertical boundary temperature gradients (which can be viewed as $P V$ 'sheets' that support vertical edge waves). While many of these may come to be viewed as geophysically relevant, this judgment should be made in the context of more generic ageostrophic instability problems for continuous flow and stratification profiles away from boundaries, as we do here. Furthermore, most of the published examples of ageostrophic instability have not been assessed from the perspective of the $A-S=0$ limit for balanced evolution, nor, as yet, has any other general criterion been identified.

In the literature of ageostrophic instability, the dynamical mechanism is often interpreted as a resonant coupling through the background shear of two neutralwave eigenmodes with coincident Doppler-shifted phase speeds, yielding a complexconjugate pair of eigenfrequencies (positive and negative imaginary parts implying exponential growth and decay). From this perspective, an ageostrophic instability is often a coupling that involves at least one mode that is essentially unbalanced such as a Kelvin or an inertia-gravity wave. In shallow-water equations Hayashi \& Young (1987) shows an unstable coupling between Kelvin and inertia-gravity waves for a shear flow on an equatorial beta-plane, and Dritschel \& Vanneste (2006) show a coupling between a balanced mode and an inertia-gravity mode for a potential vorticity front. Wang \& McWilliams (2012) show resonances between a balanced shear wave and an inertia-gravity wave or between two inertia-gravity waves in shallow-water equations on an $f$-plane with several continuous profiles for a basic flow far from boundaries. Sakai (1989) analyses Phillips' two-layer flow in a bounded channel and shows the coupling between a Rossby wave in the first layer and a Kelvin wave in the second or between two Kelvin waves. Gula, Plougonven \& Zeitlin (2009) analyse the same problem for a continuous flow, which presents a sharp shear layer in the vertical. They reproduce the coupling between waves occurring in the two-layer model. Kelvin and shear waves are coupled in a barotropic boundary current (McWilliams et al. 2004). In a centrifugally stable Taylor-Couette flow, an ageostrophic instability is shown that results from the coupling of two shear-modified Kelvin waves (Molemaker et al. 2001; Yavneh et al. 2001). In the baroclinic instability in an Eady's flow (Stone 1970; Nakamura 1988; Molemaker et al. 2005; Plougonven, Muraki \& Snyder 2005), the ageostrophic modes have a smaller scale than the geostrophic modes and are associated with a coupling between a balanced shear wave and an inertia-gravity wave near an inertia-critical layer (where the difference between the basic-state velocity and the phase speed of the wave equals the Coriolis frequency divided by the horizontal wavenumber; this layer is also a barrier for vertical propagation of neutral inertia-gravity waves). This layer differs from a classical critical layer where the phase speed equals the basic-state velocity. A critical layer is associated with geostrophic instabilities of barotropic and baroclinic basic flows (Bretherton 1966). Small-scale ageostrophic frontal instabilities are found in both layered models and laboratory experiments (Griffiths, Killworth \& Stern 1982; Griffiths \& Linden 1982; Killworth, Paldor \& Stern 1984; Pennel, Béranger \& Stegner 2012). Moore \& Peltier (1987) and Moore \& Peltier (1990) also addressed the question with the linear 
primitive equation and were able to find small-scale ageostrophic growing eigenmodes on a front with uniform potential vorticity (PV). However, a time-integration study of Snyder (1995) did not support their results. Yamazaki \& Peltier (2001) revisit the problem of a front by adding a weak cross-front horizontal gradient of PV and non-uniform stratification. They identify some small-scale instabilities, but it is not clear whether those modes are well-resolved ('The growth rates change significantly as the truncation level increases', Yamazaki \& Peltier 2001) and whether or not there are largely balanced instabilities associated with a boundary temperature gradient. Barth (1994) examines the destabilization of a front in a realistic stratification with a surface mixed layer and a surface boundary temperature gradient. He finds two types of baroclinic instability: one with a deeper vertical extent and larger horizontal scale and the other with a shallower and smaller scale. The latter may be similar to the 'mixed-layer' baroclinic instability of Boccaletti, Ferrari \& Fox-Kemper (2007).

In this paper we show that ageostrophic instability arises in a non-separable basic flow with continuous stratification far away from horizontal and vertical boundaries and with small viscosity. In $\$ \S 2-4$ we solve the problem of the linear stability of a geostrophic interior jet using both Boussinesq and a quasi-geostrophic models to assess whether modes are essentially geostrophic or not. The unstable modes are interpreted in relation to the $A-S=0$ criterion and analysed in terms of their energetics and feedback on the basic flow. The conclusions are discussed in $\S 5$.

\section{Non-dimenionalization and fluctuation equations}

We solve the linear, normal-mode stability problem for a basic-state velocity $\bar{V}(x, z)$ in geostrophic balance in a rotating, stably stratified fluid with Coriolis frequency $f>0$ and Brunt-Väisälä frequency $N$. To non-dimensionalize the governing equations, we use a horizontal scale $L$, a vertical scale $H$, and a horizontal velocity scale $V_{0}$ all based on the basic flow. The time scale is the inverse of the basic shear rate, $L / V_{0}$. Vertical velocity is scaled as $\lambda(R o / B u) V_{0}$, where $\lambda=H / L$ is the aspect ratio; $R o=V_{0} / f L$ is the Rossby number; and $B u=(N H / f L)^{2}$, the Burger number. $B u$ is also defined as $(R o / F r)^{2}$ with $F r=V_{0} / N H$ the Froude number. The background temperature field $\bar{T}^{b}(z)$ is non-dimensionalized by $T_{0}=\left(N^{2} H\right) /(g \alpha)$ with $g$ the gravitational acceleration and $\alpha$ the thermal expansion coefficient such that density is $\rho=-\rho_{0} \alpha T$. The dynamical temperature $\bar{T}$ for the basic flow and the fluctuation $T$ are scaled with $\delta T_{0}=(R o / B u) T_{0}$. The dynamical pressure field is non-dimensionalized by $(R o / B u) p_{0}$, with $p_{0}=\rho_{0} g \alpha T_{0} H=\rho_{0} N^{2} H^{2}$ the background scale. Henceforth, all quantities are non-dimensional.

The flow is governed by five non-dimensionalized numbers. The Rossby number and the Burger number are the primary parameters that we vary. The aspect ratio is kept very small at $5 \times 10^{-3}$ to characterize balanced geophysical flows. The Ekman number $E k=v / f L^{2}$, which compares the effects of viscosity $v$ and rotation, is also kept very small (usually $\sim 10^{-9}$ ) to focus on inviscid motion; it is formally retained to be able to regularize fine spatial scales in the unstable modes, if needed. Finally, the Prandtl number, $\operatorname{Pr}=\nu / \kappa$ with $\kappa$ the thermal diffusivity, is set to unity. This excludes the instability of McIntyre (1970), which requires both large $\operatorname{Pr}$ and $\lambda$ order one, hence is not relevant for oceanic meso-scale flow.

We first specify the basic flow and stratification and then define two sets of the linearized eigenmode problem for fluctuations around the basic flow: the Boussinesq equations where ageostrophic flows may arise and the quasi-geostrophic equations where only balanced flows are solutions. 

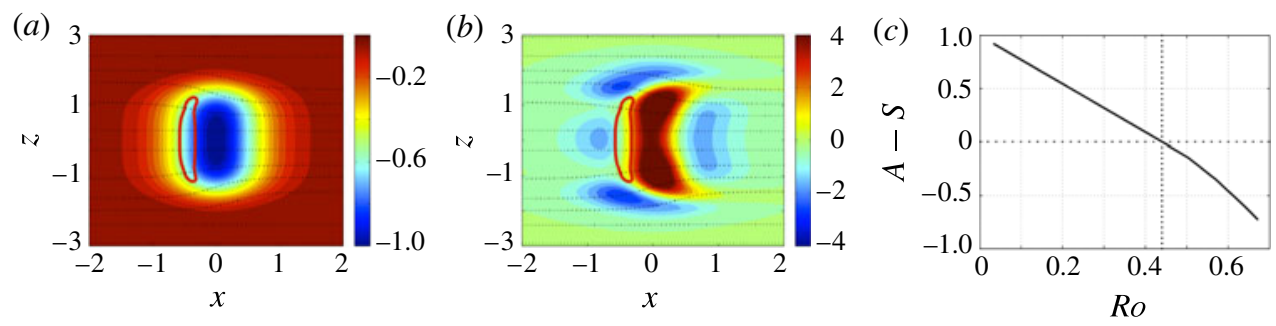

FIGURE 1. Vertical sections of $(a)$ basic-state velocity $\bar{V}(x, z)$ and $(b)$ isopycnal gradient of Ertel potential vorticity $\partial_{s} \overline{P V}(x, z)$ divided by $R o$, both for $B u=0.78$ and $R o=0.47$. Black dotted lines are basic-state isopycnals, and red lines are $A-S=0$. (c) The spatial minimum value of $A-S$ versus $R o$ for $B u=0.78$.

\subsection{The basic state}

The basic state is designated as the $(x, y, z)$ velocities plus the pressure and temperature fields, i.e. $\left\{0, \bar{V}(x, z), 0, \bar{P}(x, z), \bar{T}^{t}(x, z)\right\}$. The superscript $t$ denotes the total temperature decomposed into a background stratification $\bar{T}^{b}(z)$ and a $\bar{T}(x, z)$ field which is in thermal wind balance with $\bar{V}$ :

$$
\begin{gathered}
\bar{T}^{t}(x, z)=\bar{T}^{b}(z)+\frac{R o}{B u} \bar{T}(x, z), \\
\bar{T}_{x}(x, z)=\bar{V}_{z} .
\end{gathered}
$$

In this study we consider a simple, linear background stratification:

$$
\bar{T}^{b}(z)=\left(z-z_{b}\right)
$$

The basic-state flow that we use is an interior jet whose velocity has a central maximum with both horizontal and vertical shear, located far away from boundaries:

$$
\bar{V}(x, z)=-\exp \left(-\left(\alpha_{z} z\right)^{4}\right) /\left(\cosh \left(\alpha_{x} x\right)\right)^{2},
$$

with the particular profile coefficients $\alpha_{x}=1.49$ and $\alpha_{z}=0.66$ (figure $1 a$ ). Unstable eigenmodes with similar behaviour to those shown below are also found with different choices of $\alpha_{x}$ and $\alpha_{z}$ and with other basic flow profiles such as exp $-\left(x^{2}+z^{4}\right)$ or $\exp -\left(x^{2}+z^{2}\right)$. The basic state is not steady under diffusion, but we neglect this effect by choosing small diffusivities and a smooth basic profile.

For the parameter space we investigate here, the basic state is chosen to be gravitationally and centrifugally stable with $\bar{T}_{z}^{t}>0$ and $f \overline{P V}>0$ everywhere. $\overline{P V}$ is the Ertel potential vorticity of the basic flow, defined as

$$
\overline{P V}=\left(\operatorname{Ro} \bar{V}_{x}+1\right) \bar{T}_{z}^{t}-\operatorname{Ro} \bar{V}_{z} \bar{T}_{x}^{t} .
$$

These conditions are assured by limiting the range of values of $R o$ and $B u$.

For geostrophic fluctuations the Rayleigh-Kuo-Fjørtoft necessary condition for barotropic and baroclinic instability is that $\partial_{x} \bar{Q}$ change sign within the domain, where $\bar{Q}$ is the quasi-geostrophic potential vorticity of the basic state $(\S 2.3)$. Charney \& Stern (1962) shows that variations of $\bar{Q}$ at constant depth are approximately proportional to variations of $\overline{P V}$ along isopycnals (surfaces of constant $\bar{T}^{t}$, equivalent to isopycnal surfaces). Hoskins (1976) generalizes the necessary condition for semigeostrophic balanced flow. Eliassen (1983) explicitly implicates the sign change of 
the isopycnal gradient of potential vorticity. The importance of isopycnal potential vorticity' is extensively argued in Hoskins, McIntyre \& Robertson (1985).

We define the unit vector $s$, along the basic-state isopycnals. In terms of (2.5), the isopycnal horizontal gradient is

$$
\boldsymbol{s} \cdot \nabla \overline{P V}=\partial_{s} \overline{P V}=\partial_{x} \overline{P V}-\frac{\partial_{x} \bar{T}}{\partial_{z} \bar{T}^{t}} \partial_{z} \overline{P V} .
$$

Figure $1(b)$ illustrates $\partial_{s} \overline{P V}(x, z)$ for the basic state. We see that the sign reversal condition is well satisfied, and this continues to be true even for small Ro. Moreover, for the particular parameters $B u=0.78$ and $R o=0.47$, the minimum value of the local Richardson number $R i$, defined as $R i=B u \bar{T}_{z}^{t} / R o^{2} \bar{V}_{z}^{2}$, is 3.6, well above the threshold value for Kelvin-Helmholtz instability. In our study of ageostrophic instability, we stay within this regime of $R i>1$. Finally, figure $1(c)$ is a plot of the $(x, z)$-minimum of $A-S$ as a function of $R o$ for the basic state, with $A=1+\operatorname{Ro}\left(\bar{V}_{x}-\left(\partial_{x} \bar{T} / \partial_{z} \bar{T}^{t}\right) \bar{V}_{z}\right)$ and $S=\operatorname{Ro}\left[\left(\bar{V}_{x}-\left(\partial_{x} \bar{T} / \partial_{z} \bar{T}^{t}\right) \bar{V}_{z}\right)^{2}\right]^{1 / 2}$. For $R o>0.45$, the basic state has a non-zero area where $A-S<0$. The red line contouring $A-S=0$ is localized in the anticyclonic part of the jet, the area where the implied breakdown of balance is more likely to occur.

\subsection{Boussinesq equations}

We assume normal-mode fluctuation solutions in the form of $\{u(x, z)$, $v(x, z), w(x, z), p(x, z), T(x, z)\} \exp (\mathrm{i} l y+\sigma t)$. The downstream wavenumber is $l$. The positive real part of $\sigma$ is the unstable growth rate, and the phase speed $c_{p}$ is minus the imaginary part of $\sigma$ divided by $l$. After substituting this form into the Boussinesq equations and factoring the exponential function, we obtain the following eigenmode system:

$$
\begin{array}{r}
R o[\sigma u+\mathrm{i} l \bar{V} u]-v+p_{x}-E k \Delta^{\prime} u=0 \\
R o\left[\sigma v+\bar{V}_{x} u+\mathrm{i} l \bar{V} v+\frac{R o}{B u} \bar{V}_{z} w\right]+u+\mathrm{i} l p-E k \Delta^{\prime} v=0 \\
R o \lambda^{2}[\sigma w+\mathrm{i} l \bar{V} w]+\frac{B u}{R o} p_{z}-\frac{B u}{R o} T-\lambda^{2} E k \Delta^{\prime} w=0 \\
u_{x}+\mathrm{i} l v+\frac{R o}{B u} w_{z}=0 \\
R o\left[\sigma T+\bar{T}_{x} u+\frac{R o}{B u} \bar{T}_{z} w+\bar{T}_{z}^{b} w+\mathrm{i} l \bar{V} T\right]-\frac{E k}{P r} \Delta^{\prime} T=0
\end{array}
$$

with $\Delta^{\prime}=\partial_{x^{2}}^{2}-l^{2}+\left(\partial_{z^{2}}^{2} / \lambda^{2}\right)$ the Laplacian operator. We choose the fluctuation boundary conditions as no normal flow at the $x$ and $z$ boundaries (i.e. $u=0$ and $w=0$, respectively) and no diffusive flux of velocity and temperature through zero normal derivatives. All these conditions are conducive to small fluctuation amplitudes in the far field away from the interior jet.

\subsection{Quasi-geostrophic equations}

For the quasi-geostrophic approximation the velocity field is decomposed into a horizontal geostrophic component $\boldsymbol{u}_{g}$ and an ageostrophic component $\boldsymbol{u}_{a}$, such that $\boldsymbol{u}=\boldsymbol{u}_{g}+(R o / B u) \boldsymbol{u}_{a}$. The Boussinesq equations (2.7) are expanded in small Ro and $\lambda$ for finite $B u$ to obtain the approximate quasi-geostrophic equation (2.8) below 
as a linear differential combination of (2.7). We introduce the streamfunction $\Phi$ (where $\partial_{x} \Phi=v_{g}$ and $\partial_{y} \Phi=-u_{g}$ ), and assume a fluctuation eigenmode solution form of $\Phi(x, y, z)=\phi(x, z) \exp (\mathrm{i} l y+\sigma t)$ with quasi-geostrophic potential vorticity $q(x, z) \exp (\mathrm{i} l y+\sigma t)$. The resulting eigenmode system is

$$
\begin{array}{r}
{[\sigma q+\mathrm{i} l \bar{V} q]-\mathrm{i} l \phi\left[\bar{V}_{x x}+\frac{1}{B u} \partial_{z}\left(\frac{\bar{V}_{z}}{\bar{T}_{z}^{b}}\right)\right]-\frac{E k}{R o} \Delta_{H} q=0,} \\
{\left[\partial_{x x}^{2}-l^{2}+\frac{1}{B u} \partial_{z}\left(\frac{1}{\bar{T}_{z}^{b}} \partial_{z}\right)\right] \phi=q,}
\end{array}
$$

with

$$
\Delta_{H}=\partial_{x x}^{2}-l^{2}
$$

a horizontal Laplacian operator. Boundary conditions in $x$ and $z$ correspond to zero normal flow and zero diffusive flux. As discussed in $\S 2.1$, the relevant quasi-geostrophic potential vorticity for instability of the basic state is $\bar{Q}=\bar{V}_{x}+$ $B u^{-1} \partial_{z}\left[\bar{T} / \bar{T}_{z}^{b}\right]$, where $\bar{V}=\bar{\Phi}_{x}$ and $\bar{T}=\bar{\Phi}_{z}$.

The quasi-geostrophic model includes a diagnostic prescription of the associated $\boldsymbol{u}_{a}$ at $O(R o)$. Its instability, if any, is entirely controlled by the system (2.8), so we refer to it as a geostrophic instability, reserving the terminology ageostrophic instability for modes present in the Boussinesq model but absent in the quasi-geostrophic one.

\section{Methods}

The Boussinesq equations are discretized using standard finite differences on a twodimensional vertical staggered grid. All variables are defined at cell centres except $u$ and $w$, which are respectively defined at the midpoints of the cell horizontal edges and vertical edges. The quasi-geostrophic equations are discretized such that $\phi$ is located at cell horizontal edges. Vertical discretization is applied on a Charney \& Phillips (1953) grid: $\phi, q$ and $p$ are at the integer levels (cell centres) while $T$ is at the half-integer levels (cell bottom edges). For bottom and surface conditions, we use the Arakawa \& Moorthi (1988) discretization, following the quasi-geostrophic potential vorticity generalization of Bretherton (1966).

This yields matrix eigenvalue problems for the two fluctuation equation systems. The resulting matrices are large and sparse. For the Boussinesq equations, a spatial resolution of $150 \times 150$ grid points implies a $(112500)^{2}$ sparse matrix system. A direct method resolving the whole eigenmode spectrum was tested, but it is only feasible for very coarse grids (e.g. $20 \times 20$ ). Because the modes we find need higher spatial resolution, we investigate only the growing eigenmodes with the highest growth rates (positive real $\sigma$ ). We use the 'implicitly restarted Arnoldi method' implemented in the ARPACK software package (e.g. Arnoldi 1951; Lehoucq \& Sorensen 1996). This method, based on Krylov subspaces, computes a partial result after a relatively small number of iterations. These restrictions in grid resolution and growth rate mean that we only find spatially smooth eigenmodes without the sharp critical-layer near-singularity that could occur for small $\sigma$. These modes do not require diffusive regularization (which would act to decrease their growth rate), so we choose to use a very small value of $E k$ in the results shown. Using this method we cannot claim to know the whole eigenmode spectrum. Instead we focus on particular modes as examples of strong instability. 

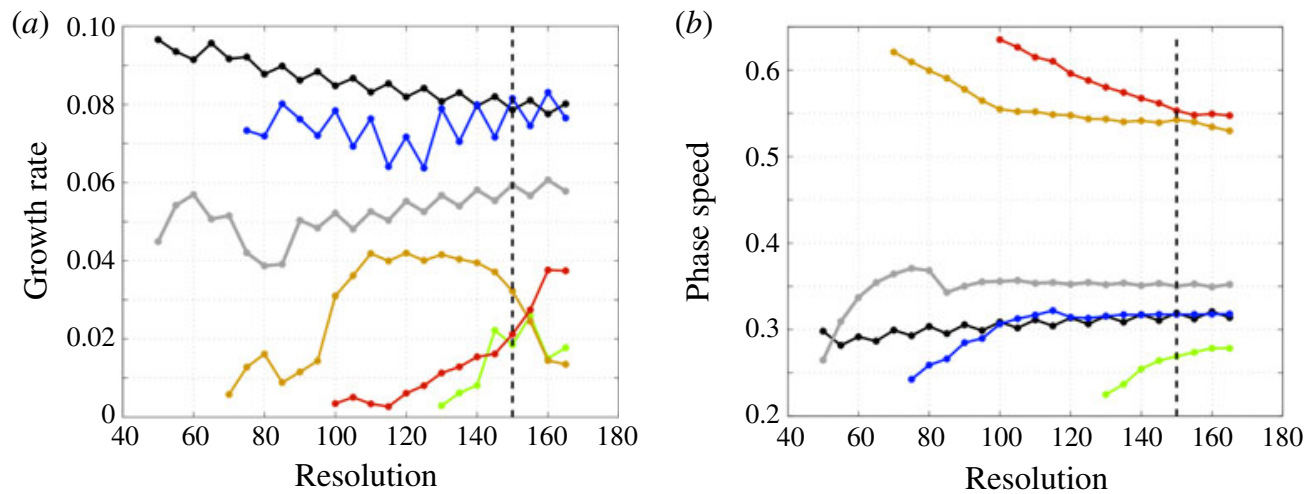

FIGURE 2. (a) Growth rate and (b) phase speed for six different eigenmodes versus $N$, where the grid size is $N \times N$. The vertical dashed line indicates the standard resolution for the results shown in subsequent figures. Convergence curves shown here are for $R o=0.47$ and $B u=0.78$ and for the three values of $l$ associated with the near-degeneracy events for different eigenmode pairs (marked with dash-dotted lines in figure $5 a$ ): black and grey curves $(l=0.77)$, red and orange curves $(l=1.52)$, blue and green curves $(l=1.4)$.

In $\S 4$ we examine a range of parameters, especially in $R o$ from small values that satisfy the assumptions for the quasi-geostrophic equations to larger ones where we expect and find departures from geostrophic balance. Scans in $R o, B u$ and wavenumber $l$ were made, using small $\lambda$ and $E k$ with $P r=1$. The geophysically most relevant ageostrophic modes are those with $B u=O(1)$. Moreover, eigenvalue convergence has been checked for grid sizes going from $50 \times 50$ to $170 \times 170$. In figure 2 are examples of convergence curves with resolution. They are calculated with fixed values of $R o$ and $B u$ at particularly interesting values of $l$ corresponding to the near-degeneracy event that occurs for the three pairs of unstable modes (discussed in $\S 4$ ). For these modes we note that the eigenvalues with non-dimensional growth rates $\sigma$ higher than 0.05 are not far from convergence at the resolution of $150 \times 150$ (i.e. their phase speed and growth rates have reached an asymptotic limit in grid number $N$ ). Curves for smaller eigenvalues are not yet well converged. We use this level of $\sigma$ as a rough guide to computational accuracy for the modes of interest, indicated by a shaded area in figures 3 and 5. Nevertheless, we do track some of the modes below this level as they emerge and develop toward larger $\sigma$ values with changes in $R o$ and $l$. We have checked that the associated eigenmode shapes do not change greatly whether $\sigma$ has converged or not.

\section{Unstable eigenmodes}

\subsection{Quasi-geostrophic and Boussinesq eigenmodes versus Ro}

The Boussinesq eigenvalue problem is first solved for a range of $R o$ values with fixed order-one values of $B u=0.78$ and $l=1.19$ (figure 3). We trace each mode's growth rate and phase speed to obtain smoothly continuous curves for both those quantities. In the quasi-geostrophic limit $(R o \rightarrow 0)$, only geostrophic eigenmodes have a finite positive growth rate. At small $R o$, Boussinesq eigenmodes are expected to be close to these quasi-geostrophic modes, and this is confirmed for the three modes analysed in figure 3. Furthermore, figure 4 compares $u$ and $v$ eigenfunction patterns of the Boussinesq 'black' mode (black curve in figure 3 at small Ro) to its quasi- 

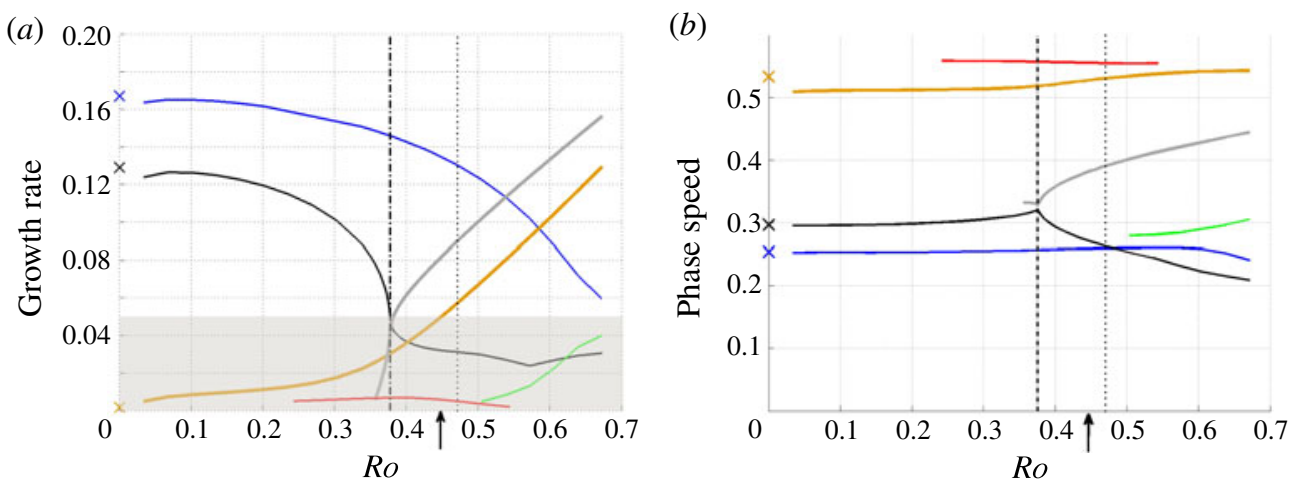

FIGURE 3. (a) Growth rate and (b) phase speed versus Ro for several Boussinesq eigenmodes coded by colour. Cross-marks at $R o=0$ are for the equivalent quasi-geostrophic eigenmodes. This plot is for $B u=0.78$ and $l=1.19$. The arrow marks the $R o$ value where $\min (A-S)$ changes sign, and the black dash-dotted line indicates the near-degeneracy point for the 'black-grey' mode pair (zoomed in figure 6). The shaded area is a guide to the computational accuracy $(\S 3)$. The black dotted vertical line at $R o=0.47$ is where an intersecting parameter survey in $l$ values is made in figure 5 .

geostrophic equivalent. To aid this structural comparison, the modes are given a common amplitude and $(y, t)$-phase normalization. The eigenfunction patterns are very similar, corroborating the equivalence of the unstable mode in this limit of $R o \rightarrow 0$. In this quasi-geostrophic limit the mode realizes the permitted reflection symmetries relative to the $x$ and $z$ axes, but we will see (figure 7) that the former symmetry is lost at finite Ro. The importance of the critical surface where $\bar{V}(x, z)=c_{p}$ is clear as a zone of high gradient at the edge of the eigenmode in $v$. An inertia-critical surface where $\bar{V}=c_{p} \pm 1 /(l R o)$ cannot occur for small Ro and finite $l$ and $c_{p}$.

The two strongly unstable geostrophic modes shown above ('black' and 'blue') initially show little change in growth rate and $c_{p}$ with increasing $R o$, but a weakly unstable geostrophic mode ('orange') amplifies strongly with Ro and several new modes arise at finite $R o$ (figure 3). The latter appear in the neighbourhood of the violation of the $A-S>0$ balanced-integrability criterion represented by an arrow on the abscissa. Two of the modes ('grey' and 'green') have no equivalent in quasigeostrophic dynamics; hence, these modes are inherently ageostrophic. Furthermore, figure 3 illustrates a sharp transition for the 'black' and 'grey' modes at a critical $R o \approx 0.38$, where they have similar phase speeds and growth rates. Here, two initially distinct, unstable modes almost coalesce into two degenerated eigenmodes as the control parameter Ro varies. Beyond this transition the dispersion curves diverge. This phenomenon of near-degeneracy is also described in Salwen \& Grosch (1972) and Salwen, Cotton \& Grosch (1980) for a Poiseuille flow in a circular pipe; Craik (1988) reports these examples as a 'near-bifurcation' where dispersion curves almost meet and where modes may exchange their identities. However, the term bifurcation is commonly reserved for a change in the state of the fluid flow which is not obviously the case here. Consequently, we will use the terminology 'near-degeneracy'. We analyse this event further in $\S 4.2$.

A different parameter scan is made in the along-front wavenumber $l$ for fixed values of $B u=0.78$ and $R o=0.47$, with the resulting growth rate and phase speed curves shown in figure 5 for the same Boussinesq eigenmodes as before (figure 3). The 
(a)

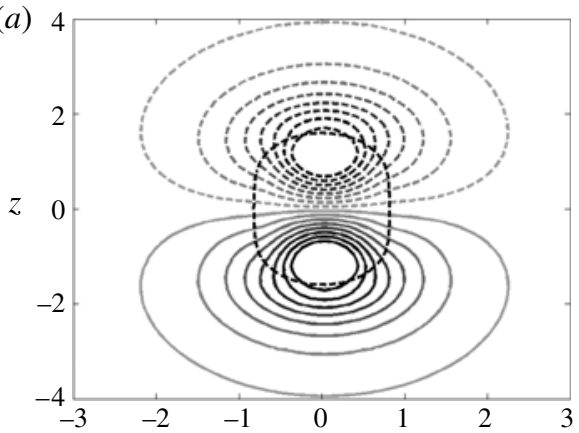

(c)

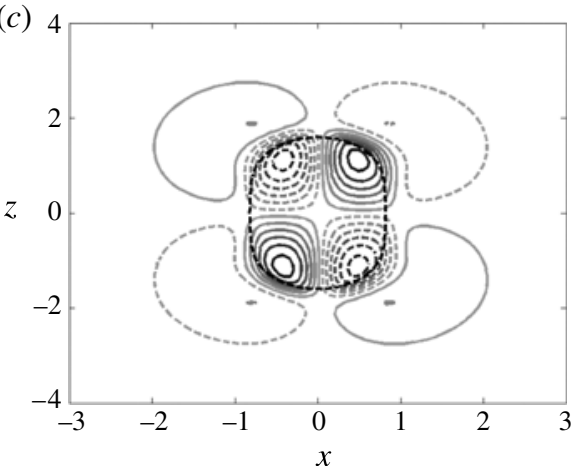

(b)

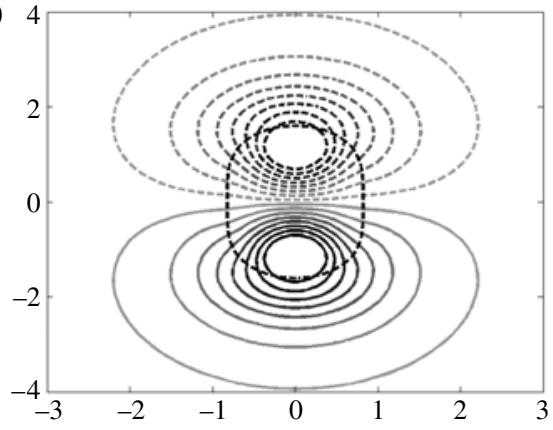

(d)

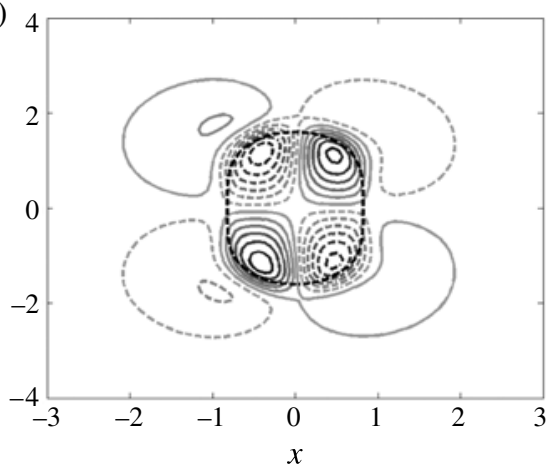

FIGURE 4. Vertical sections of normalized eigenfunctions $u(x, z)(a, b)$ and $v(x, z)(c, d)$ for the 'black' mode, in $(a, c)$ quasi-geostrophic and $(b, d)$ Boussinesq equations at $R o=0.03$. Contours are every 0.12 from -0.9 to 0.9 (solid positive, dashed negative). The bold dashed line is the location of the critical surface where $\bar{V}=c_{p}$, where $c_{p}$ is the phase speed for the mode. This plot is for $B u=0.78$ and $l=1.19$.
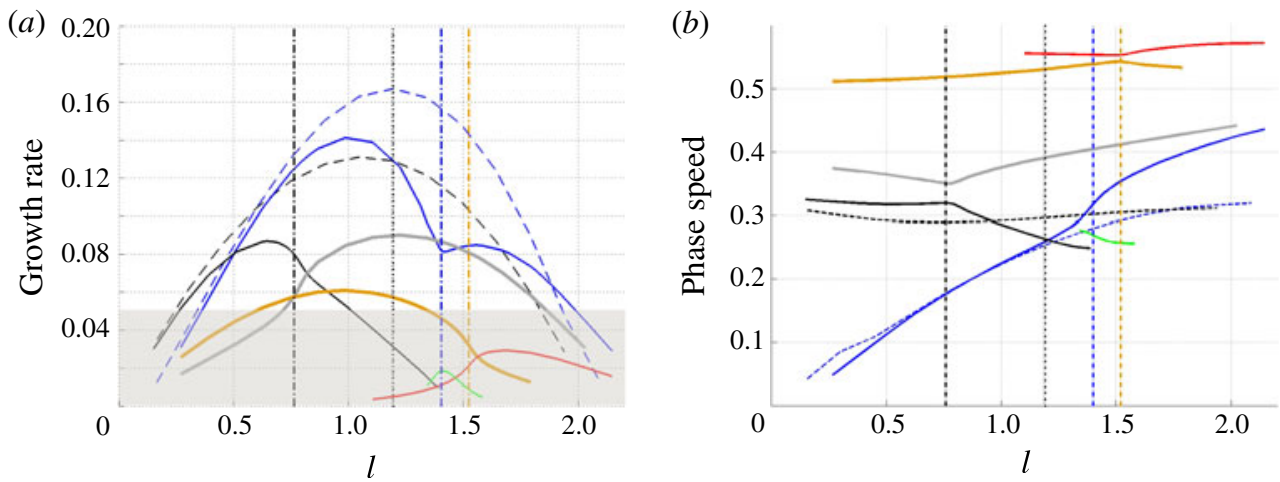

FIGURE 5. (a) Growth rate and (b) phase speed versus $l$ for several eigenmodes with $R o=0.47$ and $B u=0.78$ : Boussinesq (solid lines) and quasi-geostrophic (dashed). The black dotted line at $l=1.19$ coincides with the $R o$ section in figure 3 , and the eigenmode colour scheme here is the same. The dash-dotted lines indicate near-degeneracy points, and the shaded area is again a guide to the computational accuracy $(\S 3)$. 

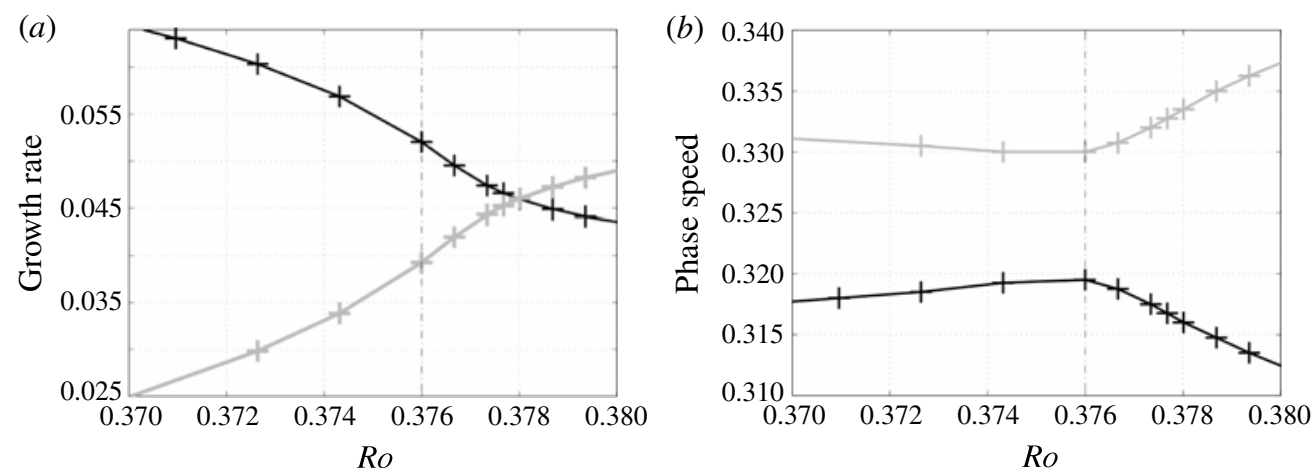

FIGURE 6. (a) Growth rate and $(b)$ phase speed in a zoom of the near-degeneracy neighbourhood of Boussinesq 'grey' and 'black' modes in figure 3, with the vertical line here at the same $R o=0.376$ as the dash-dotted line there. Plus symbols indicate the data points in the control parameter $R o$.

dispersion curves for the equivalent quasi-geostrophic modes are also plotted with dashed curves for the 'black' and 'grey' modes. The dispersion curves match closely between the two models at small Ro (not shown). Here at finite Ro, the models differ substantially, especially for larger $l$ after the occurrence of near-degeneracy events in the Boussinesq equations, both between 'black' and 'grey' modes (as before) and between 'blue' and 'green' modes. Additional ageostrophic instability modes appear in the large- $l$ region with relatively weaker growth rate.

\subsection{A nearly-degenerate Boussinesq eigenmode pair}

We now focus on the 'black' and 'grey' pair of eigenmodes. Figure 6 is a zoom-in on figure 3, showing the near-degeneracy as a continuous transition of the two modes, i.e. without discontinuity in the growth rate and phase speed curves. Growth rate curves cross at a $R o$ value close to the point where the phase speeds are close but themselves do not cross. Note that the data points on the curves indicate the resolution in Ro, which is fine enough to be certain of the tracking of the separate modes through the event.

The spatial structure of the 'black' and 'grey' eigenfunctions is shown in figure 7 for a $R o$ value (0.370) just before the crossing of the growth curves at $R o=0.378$, close to the near-degeneracy event. The velocity field of the 'black' mode (figure 7a,c) is more confined about the critical surface $\left(\bar{V}=c_{p}\right)$ than it was at the smaller $R o$ value (figure $4 b, d$ ), and its structure is now quite close to that of the emerging 'grey' mode that it is pairing with (figure $7 b, d$ ). This is further manifestation of the near-degeneracy of the mode pair. Both modes manifest a right-left asymmetry, in contrast to the small-Ro geostrophic mode in figure $4(b, d)$. We have checked that the location of the inertia-critical surface $\left(\bar{V}=c_{p} \pm 1 /(l R o)\right)$ does not occur within the domain for these modes, so it cannot be considered relevant. This is in contrast to ageostrophic baroclinic instability in the Eady flow $(\S 1)$, and we show in $\S 4.3$ that the near-degeneracy transition is a result of vertical shear instability and Reynolds stress energy conversion rather than baroclinic conversion of potential energy.

The degree of eigenmode ageostrophy is illustrated in figure 8 for the relative magnitude of the horizontally divergent component of the horizontal velocity, $\Delta=$ $\left|\boldsymbol{u}_{\text {div }}\right| /|\boldsymbol{u}|$, in a decomposition into horizontally divergent and rotational (i.e. carrying 
(a)

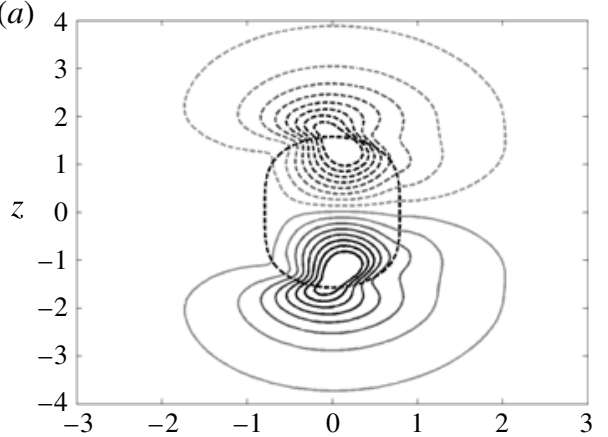

(c)

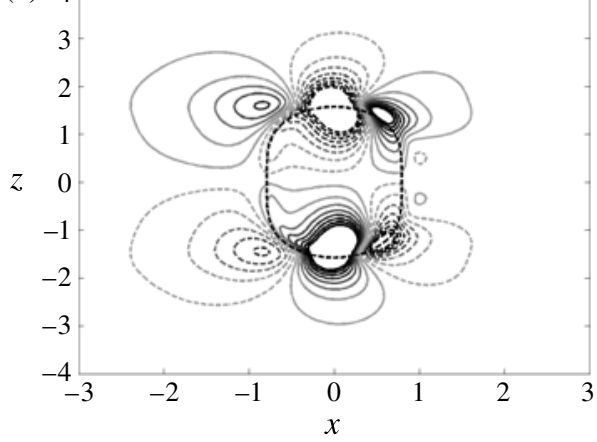

(b)

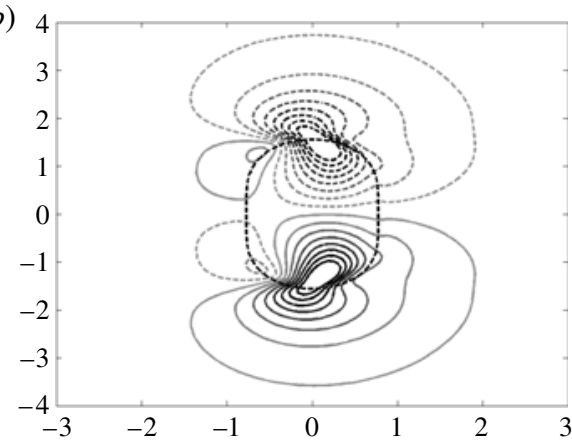

(d)

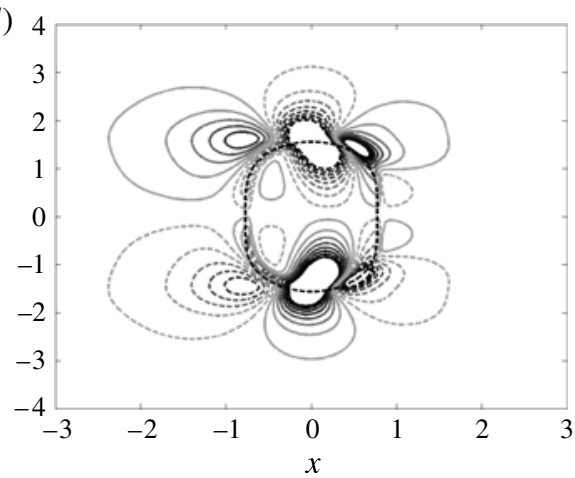

FIGURE 7. Vertical sections of normalized eigenfunctions $u(x, z)(a, b)$ and $v(x, z)(c, d)$ for the Boussinesq eigenmodes: $(a, c)$ 'black' and $(b, d)$ 'grey'. The parameters are for the neardegeneracy event: $B u=0.78, R o=0.37$ and $l=1.19$. The bold dotted line is the location of the critical surface, $\bar{V}=c_{p}$.
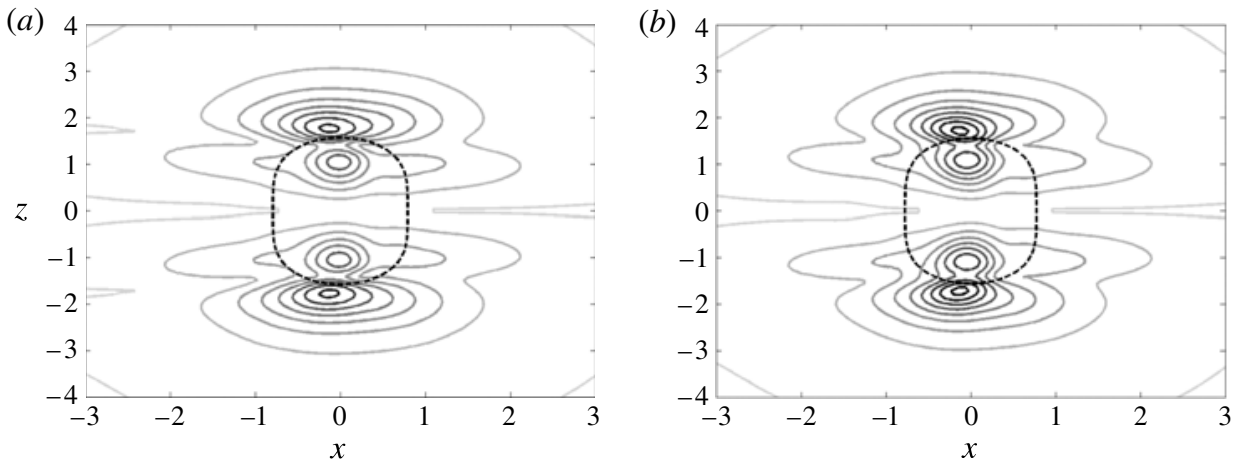

FIGURE 8. Vertical sections of $\Delta(x, z)$, the local fraction of the divergent component in the horizontal velocity field: $(a)$ 'black' mode and $(b)$ 'grey' mode. $B u=0.78, R o=0.37$ and $l=1.19$. The maximum amplitude is 0.18 with a contour interval 0.022 . The bold dashed line shows the location of the critical surface. 


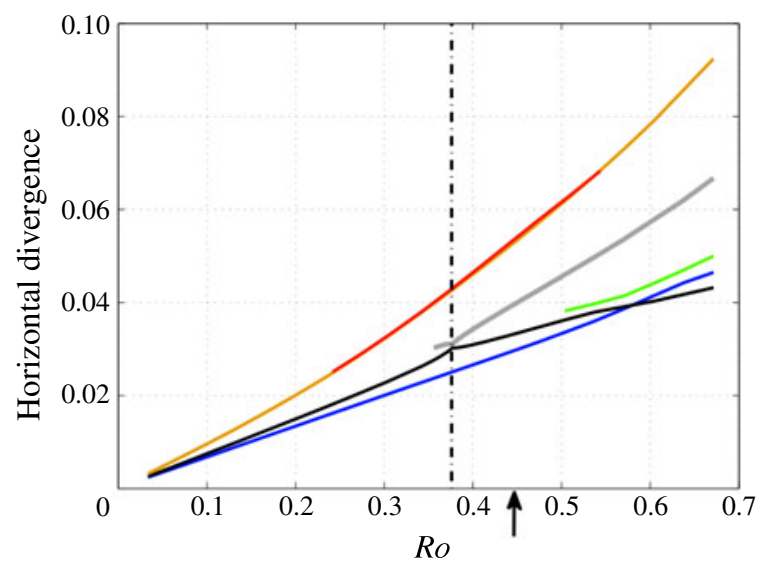

FIGURE 9. Domain-averaged fraction of the divergent component of the horizontal velocity field, $\delta$, for the same eigenmodes as in figure 8 . The arrow marks where $\min (A-S)$ changes sign, and the vertical line is at $R o=0.376$ (as in figures 3 and 6).

the vertical component of relative vorticity) parts, $\boldsymbol{u}=\boldsymbol{u}_{d i v}+\boldsymbol{u}_{r o t}$. In quasi-geostrophic theory, $\Delta$ is vanishingly small with $R o$. At finite $R o$ the Boussinesq eigenmodes have large $\Delta$ around the critical surface. The domain average of the horizontally divergent part is plotted versus $R o$ in figure 9. We define $\delta=\left\langle\left|\boldsymbol{u}_{d i v}\right|\right\rangle_{D} /\langle|\boldsymbol{u}|\rangle_{D}$ with $\langle\cdot\rangle_{D}$ an average over the domain. As expected, eigenmodes with a quasi-geostrophic counterpart have vanishing $\delta$ at small Ro. With increasing Ro, $\delta$ increases continuously indicating the growth of an ageostrophic velocity component. At finite $R o$, the emerging ageostrophic modes ('grey' and 'green') have higher horizontal divergent parts than the eigenmodes they are pairing with (respectively, the 'black' and 'blue' modes). The critical Ro for the near-degeneracy event is co-located in this graph with a kink in the curves where the mode-pair $\delta$ values intersect. We stress the fact that, in the parameter region where the near-degeneracy occurs, the pairing eigenmodes are very similar in dispersion curves (figure 6), eigenmode patterns (figure 7), and the rotational and divergent parts of their velocity fields. After the near-degeneracy event, the increase with $R o$ of the horizontally divergent fraction continues in all the eigenmodes.

\subsection{Energetics}

A diagnostic perspective on unstable eigenmodes is the nature of the energy conversion from the basic flow to the growing fluctuations. We analyse the volumeintegrated energetics of eigenmodes in the limit of small fluctuation amplitude (e.g. Gill 1982). These are derived from $y$-averaged evolution equations for the kinetic energy density, $\mathrm{Ke}$, and the available potential energy density, $\mathrm{Pe}$, defined by

$$
\begin{aligned}
& K e=\frac{u u^{\star}+v v^{\star}}{2}, \\
& P e=\frac{T T^{\star}}{2 B u \bar{T}_{z}^{b}} .
\end{aligned}
$$

The star denotes the complex conjugate. 


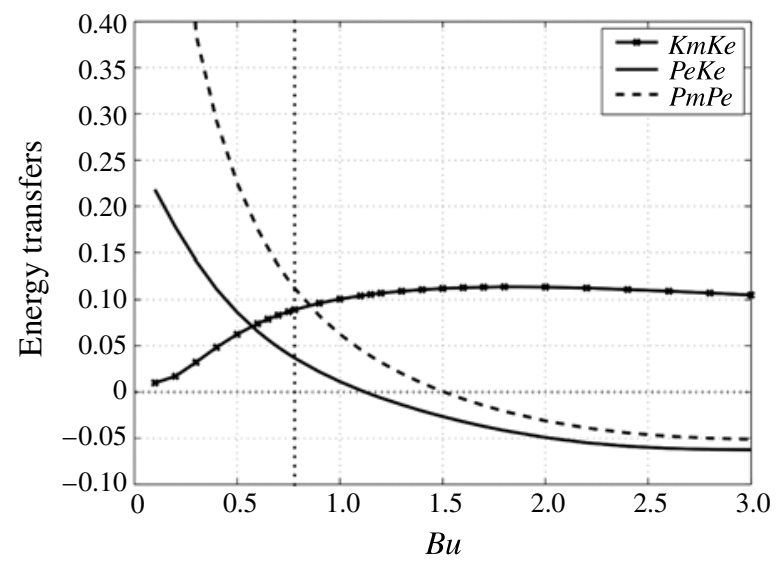

FIGURE 10. Quasi-geostrophic energy conversions versus $B u$ for the 'black' mode at $l=1.19$. This is the asymptotic result as $R o \rightarrow 0$. The vertical line marks $B u=0.78$.

\subsubsection{Quasi-geostrophic energy conversion}

In the quasi-geostrophic equations, $K e$ is defined with the geostrophic components of velocity, and the $y$-averaged fluctuation energy evolution equations are

$$
\begin{aligned}
& \operatorname{Ro}[2 \sigma\langle K e\rangle+\underbrace{\bar{V}_{x}\left\langle u_{g} v_{g}^{\star}\right\rangle}_{- \text {KmKe }}-\underbrace{\frac{1}{B u}\left\langle w_{a} T^{\star}\right\rangle}_{\text {PeKe }}+\underbrace{\frac{1}{B u} \nabla \cdot\left(\left\langle\boldsymbol{u}_{a} \phi^{\star}\right\rangle\right)}_{\text {Press }}]=\underbrace{E k\left\langle\left[u_{g}^{\star} \Delta_{H} u_{g}+v_{g}^{\star} \Delta_{H} v_{g}\right]\right\rangle}_{\text {Diss }} \\
& R o[2 \sigma\langle P e\rangle+\underbrace{\frac{\bar{T}_{x}}{B u \bar{T}_{z}^{b}}\left\langle u_{g} T^{\star}\right\rangle}_{-P m P e}+\underbrace{\frac{1}{B u}\left\langle w_{a} T^{\star}\right\rangle}_{\text {PeKe }}]=\underbrace{\frac{E k}{P r B u \bar{T}_{z}^{b}}\left\langle T^{\star} \Delta_{H} T\right\rangle}_{\text {Diss }} ;
\end{aligned}
$$

$\langle\cdot\rangle$ denotes an average over the $y$-direction. For the small $E k$ values we have used, the dissipation terms Diss are negligible for the eigenmodes we are focusing on. In a volume integral the pressure work Press is zero with our boundary conditions. The remaining terms are $\mathrm{PmPe}, \mathrm{PeKe}$ and $\mathrm{KmKe}$, which convert energy, respectively, from available potential energy of the basic-state flow to fluctuation available potential energy, from fluctuation available potential energy to fluctuation kinetic energy, and from basic-flow kinetic energy to fluctuation kinetic energy.

We characterize the geostrophic instability type by the relative importance of $P m P e$ and $K m K e$, i.e. baroclinic instability associated with the vertical shear $\bar{V}_{z}$ and horizontal buoyancy flux (i.e. $\langle u T\rangle \neq 0$ ) or barotropic instability associated with the horizontal shear $\bar{V}_{x}$ and horizontal Reynolds stress (i.e. $\langle u v\rangle \neq 0$ ). Their volume averages are evaluated as a function of $B u$, normalized by the kinetic energy average, and plotted in figure 10. Small $\mathrm{Bu}$ indicates a strong vertical shear compared to the horizontal shear in the basic flow.

At small $B u(B u<0.8)$, the main source of instability is baroclinic conversion of available potential energy (positive $P m P e$ and $P e K e$ ). For $B u>0.8$, the main source of instability is a barotropic conversion of kinetic energy (positive $\mathrm{KmKe}$ ). 


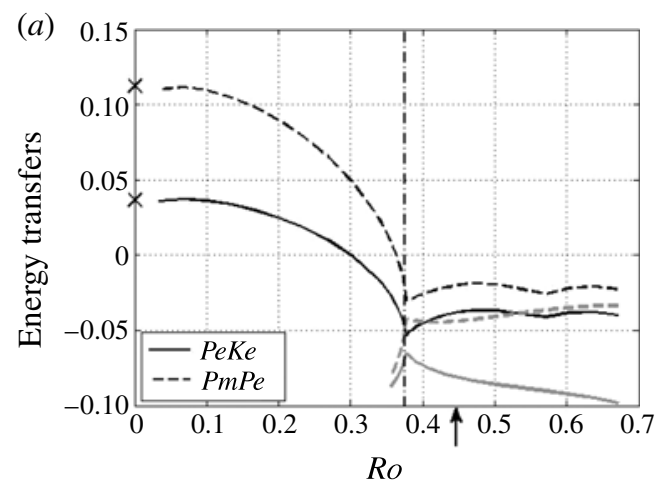

(b)

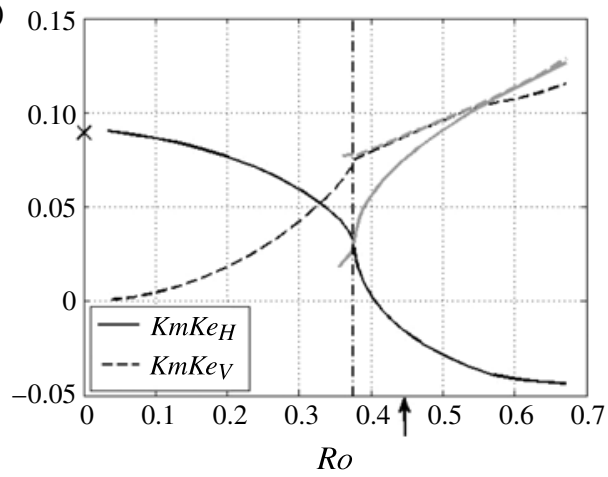

FIGURE 11. Boussinesq energy conversions versus Ro for 'black' and 'grey' modes. Crossmarks are for the equivalent quasi-geostrophic eigenmodes as on figure 3. The vertical dashdotted line marks the near-degeneracy event at $R o=0.376$, and the arrow marks where $\min (A-S)$ changes sign. By chance $K m K e_{H} \approx K m K e_{V}$ for the 'grey' mode for $R o \geqslant 0.55$ where their curves overlap. $B u=0.78$ and $l=1.19$.

At $B u \sim 1.5, P m P e$ crosses zero, indicating that the fluctuation displacements align with the basic-state isopycnals, and for larger $B u$ it is even a sink of fluctuation energy. The cases illustrated in figures 3-9 are for $B u=0.78$, where the flow has a mixed baroclinic-barotropic instability with $\mathrm{PmPe}$ and $\mathrm{KmKe}$ both positive.

\subsubsection{Boussinesq energy conversion}

In the Boussinesq equations the $y$-averaged fluctuation energy evolution equations are

$$
\begin{gathered}
\operatorname{Ro}[2 \sigma\langle K e\rangle+\underbrace{\bar{V}_{x}\left\langle u v^{\star}\right\rangle}_{-K m K e_{H}}+\underbrace{\frac{R o}{B u} \bar{V}_{z}\left\langle v^{\star} w\right\rangle}_{-K m K e_{V}}-\underbrace{\frac{1}{B u}\left\langle w^{\star} T\right\rangle}_{\text {PeKe }}]+\underbrace{\left\langle u v^{\star}-u^{\star} v\right\rangle}_{\text {Coriolis }} \\
+\underbrace{\nabla \cdot\left(\left\langle\boldsymbol{u}^{\star} p\right\rangle\right)}_{\text {Press }}=\underbrace{\left.E k\left\langle u^{\star} \Delta^{\prime} u+v^{\star} \Delta^{\prime} v+\lambda^{2} \frac{R o^{2}}{B u^{2}} w^{\star} \Delta^{\prime} w\right]\right\rangle}_{\text {Diss }}, \\
\operatorname{Ro}[2 \sigma\langle\text { Pe }\rangle+\underbrace{\frac{\bar{T}_{x}}{B u \bar{T}_{z}^{b}}\left\langle u T^{\star}\right\rangle+\frac{R o \bar{T}_{z}}{B u^{2} \bar{T}_{z}^{b}}\left\langle w T^{\star}\right\rangle}_{-P m P e}+\underbrace{\frac{1}{B u}\left\langle w T^{\star}\right\rangle}_{\text {PeKe }}]=\underbrace{\frac{E k}{P r} \frac{1}{B u \bar{T}_{z}^{b}}\left\langle T^{\star} \Delta^{\prime} T\right\rangle}_{\text {Diss }} .
\end{gathered}
$$

Again, the Diss terms are negligible, and Press has zero domain average. The Coriolis term is purely imaginary and does not have an effect on the growth rate of the fluctuation. The remaining terms are the same kinds of conversion as in (4.2). However, the conversion from basic-flow kinetic energy to fluctuation kinetic energy has both a horizontal part $K m K e_{H}$ and a vertical part $K m K e_{V}$, whose sum is the total $K m K e$. In the quasi-geostrophic limit, $K m K e_{V}$ does not contribute at leading order in $R o$, so $K m K e=K m K e_{H}$.

Energy conversions averaged over the domain and normalized by the kinetic energy average are plotted in figure 11 for a range of $R o$ values at $B u=0.78$, again focusing 
on the 'black' and 'grey' eigenmode pair. At small $R o$ and finite $B u$, the 'black' mode is, as in its quasi-geostrophic equivalent, a mixture of geostrophic baroclinic $(\mathrm{PeKe}>0)$ and horizontal-barotropic $\left(\mathrm{KmKe}_{H}>0\right.$ in figure 11b) instabilities. PmPe is maximum as $R o \rightarrow 0$, indicating the importance of baroclinic geostrophic instability. As $R o$ increases the baroclinic conversion of available potential energy drops and becomes negative, as does the PeKe conversion, while kinetic energy conversions from the basic flow to the fluctuation increase (especially $K m K e_{V}$ ). The companion 'grey' mode has no source of energy coming from baroclinic conversion (figure 11a). It grows only through a conversion from kinetic energy of the basic flow to kinetic energy of the fluctuation (figure $11 b$ ). Thus, with increasing $R o$, the instability type for those two modes progressively changes into a shear instability with large $\mathrm{KmKe}_{V}>0$; this is an inherently ageostrophic process associated with vertical Reynolds stress (i.e. $\langle u w\rangle \neq 0)$. The near-degeneracy event is evident in an abrupt change of the energy conversion curves in the neighbourhood of $A-S=0$.

\subsection{Isopycnal homogenization tendency for Ertel potential vorticity}

As a complement to the integrated energy conversion view of the interaction of the basic flow and the unstable Boussinesq eigenmodes ( $\$ 4.3 .2)$, we now make a local analysis of the relation between the $y$-averaged flux of fluctuation Ertel potential vorticity $(p v)$ and $\overline{P V}(x, z)$ in the basic flow. We decompose the total potential vorticity into its $y$-average, $\langle P V\rangle(x, z, t)$ which may change due to the unstable fluctuations, and the fluctuation $p v$ defined by

$$
p v=\left(\frac{\lambda^{2} R o^{2}}{B u} \mathrm{i} l w-R o v_{z}\right) \bar{T}_{x}^{t}-\frac{R o^{2}}{B u} \bar{V}_{z} T_{x}+R o\left(v_{x}-\mathrm{i} l u\right) \bar{T}_{z}^{t}+\frac{R o}{B u}\left(1+R o \bar{V}_{x}\right) T_{z} .
$$

Initially, before the fluctuations grow to a significant amplitude, $\langle P V\rangle \approx \overline{P V}$. Subsequently, the fluctuations will act to modify the evolution of $\langle P V\rangle$ according to the following balance relation with quadratic flux divergences in the fluctuation fields:

$$
\partial_{t}\langle P V\rangle+\langle\boldsymbol{U}\rangle \cdot \nabla\langle P V\rangle=-\nabla \cdot \boldsymbol{F}\{P V\},
$$

where the flux vector in the $(x, z)$ plane is $\boldsymbol{F}=\left\{F^{x}, F^{z}\right\}$ with components,

$$
\begin{aligned}
& F^{x}\{P V\}=\frac{1}{2} \operatorname{Re}\left(\left\langle u p v^{\star}\right\rangle\right), \\
& F^{z}\{P V\}=\frac{R o}{2 B u} \operatorname{Re}\left(\left\langle w p v^{\star}\right\rangle\right) .
\end{aligned}
$$

We next project $\boldsymbol{F}$ onto locally isopycnal and diapycnal directions, with unit vectors $\boldsymbol{s}$ and $\boldsymbol{n}$ which are tangential and normal, respectively, to the basic-state isopycnal surfaces in $\bar{T}^{t}(x, z)$. We define isopycnal and diapycnal eddy diffusivities, $\kappa^{s}$ and $\kappa^{n}$ by the flux-gradient relations,

$$
\begin{aligned}
\boldsymbol{s} \cdot \boldsymbol{F}\{P V\} & =-\kappa^{s} \partial_{s}\langle P V\rangle, \\
\boldsymbol{n} \cdot \boldsymbol{F}\{P V\} & =-\kappa^{n} \partial_{n}\langle P V\rangle .
\end{aligned}
$$

Because in the mean flow evolution (equation (4.5)), only divergence of the flux matters, we remove the $y$-rotational component for the vector $\boldsymbol{F}\{P V\}$ in the cross-flow plane, although it is relatively small compared to the divergent component that we show. 
(a)

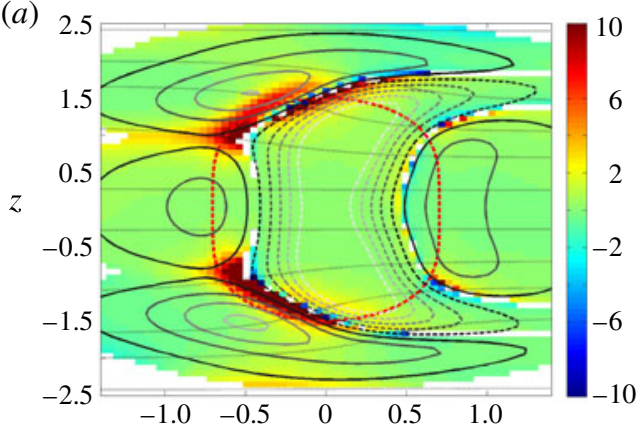

(c) 25

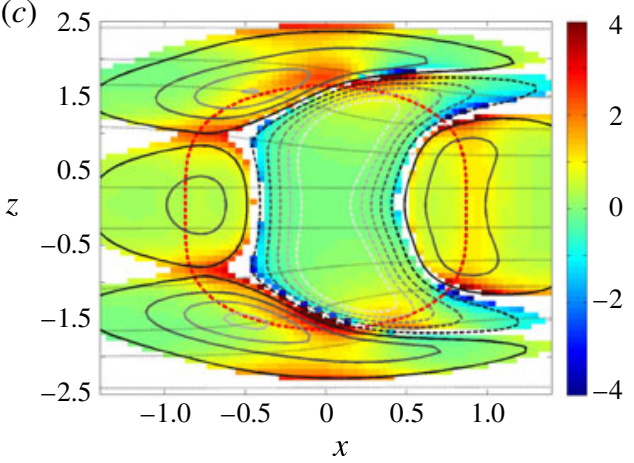

(b)

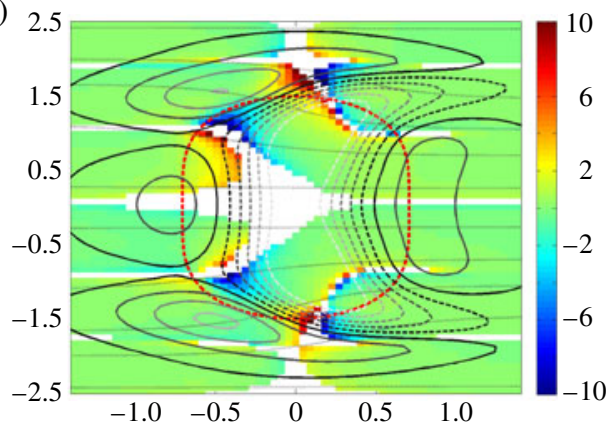

(d)

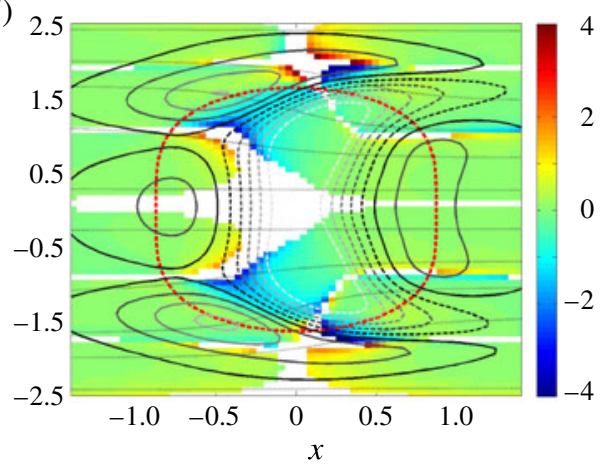

FIGURE 12. Vertical sections of $(a, c) \kappa^{s}(x, z)$ and $(b, d) \kappa^{n}(x, z)$ with colour shading for $(a, b)$ 'grey' and $(c, d)$ 'blue' Boussinesq eigenmodes at $R o=0.47, B u=0.78$ and $l=1.19$. The black contours (positive solid, negative dashed) are the basic-state potential vorticity isopycnal gradient: $\partial_{s} \overline{P V}$. Colouration of $\kappa$ is suppressed where the corresponding gradient of $\overline{P V}$ is very small. The dashed red line is the location of the critical layer, and horizontal dotted lines are contours of the basic stratification $\bar{T}^{t} . \kappa^{s}$ is positive in the vicinity of the conjunction of the critical surface and the sign change in $\partial_{s} \overline{P V}$.

An arguably better approach to estimate an eddy diffusivity of PV would have been to remove the skew component of PV flux (i.e. the component normal to the mean gradient of PV) which is advective in nature (Plumb \& Ferrari 2005). Following the transformed Eulerian-mean (TEM) formalism, we have estimated the residual PV flux (i.e. raw flux minus its skew component, not shown here). We do see differences between residual flux and the divergent component of raw flux, but mainly in areas where the mean isopycnal PV gradient vanishes. Indeed, it is possible for raw PV flux not to vanish even when the mean isopycnal gradient of PV vanishes, while residual PV flux vanishes. But, in our study, the mean isopycnal gradient of PV vanishes very locally at the edge of the jet. Thus, the residual PV flux vanishes only in confined area, and the relative importance of the skew component is not too strong and the main pattern of PV flux does not change. Hence, we choose to show in this paper the simpler PV analysis in (4.5)-(4.7) rather than the more elaborate TEM analysis.

We make this analysis for two unstable eigenmodes with parameters well beyond their near-degeneracy events, where the divergence amplitude $\delta$ and $\mathrm{KmKe}_{V}$ energy conversions clearly indicate ageostrophic behaviour (i.e. for $R o=0.47$ in figure 12). This case is also beyond the violation of the $A-S>0$ balanced-integrability condition (cf. figure 1). The spatial distributions of the diagnosed $\kappa$ fields are non-uniform and 
not everywhere positive. The TEM analysis mentioned before corrects the few negative values of $\kappa$ into positive values. Nevertheless, $\kappa_{s}$ calculated here has large, positive values in the vicinity of the critical surface (as predicted by Lindzen, Farrell \& Tung 1980 in the quasi-geostrophic limit). Furthermore, $\kappa^{s}>0$ is largest around the sign change of the isopycnal potential vorticity gradient $\partial_{s} \overline{P V}$, indicating the satisfaction of the Rayleigh necessary condition for instability $(\$ 2.1)$. The positive value is consistent with the fluctuations being created by the destabilization of the basic flow and then acting to counteract the cause of its growth by diminishing the magnitude of $\partial_{s}\langle P V\rangle$ where it is largest in the neighbourhood of the critical surface. This is consistent with the possibility of equilibration between the $y$-averaged flow and fluctuations at finite amplitude (not shown here) toward a reduced $P V$ gradient, sometimes referred as homogenization. The tendency toward $P V$-homogenization along basic-state isopycnals is also shown in Kuo, Plumb \& Marshall (2005) and Cerovečki, Plumb \& Heres (2009) for a baroclinic instability example. The diapycnal diffusivity $\kappa^{n}$ in figure 12 is smaller than $\kappa^{s}$ and less generally positive, so we consider it as indicating a secondary effect on $\langle P V\rangle$ without a ready interpretation.

Finally, notice that the amplitudes of both $\partial_{s} \overline{P V}$ and $\kappa^{s}$ are larger on the left side of $\bar{V}$ in figure 12, as are the eigenmodes (not shown), which is the anticyclonic side where $0<\overline{P V}<\bar{T}^{b}=1$ and $A-S<0$ in figure 1. This supports a characterization of the instability beyond the near-degeneracy event as an anticyclonic ageostrophic in its type.

\section{Conclusions and discussion}

We solve the linear stability problem of an interior two-dimensional jet $\bar{V}(x, z)$ with both horizontal and vertical shear in a continuously stratified, rotating, Boussinesq flow. We examine a range of small and intermediate values of $R o$ where the balanced basic flow is stable to unbalanced instabilities (i.e. gravitational, centrifugal and Kelvin-Helmholtz) known to occur at even larger Ro. The basic flow satisfies the Charney \& Stern (1962) necessary condition for geostrophic baroclinic and barotropic instability. Indeed, geostrophic eigenmodes are found, and at small $R o$ the Boussinesq eigenmodes have equivalent quasi-geostrophic eigenmodes. In addition to these expected geostrophic instabilities, we demonstrate the existence at finite $R o$ of ageostrophic eigenmodes that have no counterpart in the companion quasi-geostrophic model and that exhibit growth rates as large as the geostrophic ones. For all unstable modes, discernible departures from geostrophic balance occur at finite $R o$ values.

In the examples presented here, the attainment of significant growth rate for an unstable ageostrophic mode - comparable to that of the geostrophic modes - coincides with a near-degeneracy event between pairs of unstable modes with nearly coincident phase speeds and structurally similar eigenfunction patterns. The near-degeneracy events occur for $R o$ values that are in the neighbourhood of the condition $A-S=0$, hence they can be viewed as anticyclonic, ageostrophic in type, similar to previous examples for more limited basic flows. This supports the conjecture that the limits to integrability of balance equations may coincide with the onset of unbalanced evolution (as is known for other integrability criteria associated with gravitational and centrifugal instability; McWilliams et al. 1998). Unlike a common interpretation of ageostrophic instability as a shear-assisted resonance of two neutral modes near marginal instability $(\S 1)$, the near-degeneracy event is between two unstable modes well away from either one's marginal instability. 
The characteristics of the modes change as parameter values vary across the neardegeneracy event. At parameter values before a near-degeneracy event, the instabilities are either geostrophic or have a very weak growth rate (consistent with growth rates that are exponentially small, $\sigma \sim \exp (-1 / R o)$ as $R o \rightarrow 0$, for the ageostrophic modes in Vanneste \& Yavneh 2007). In our examples, the quasi-geostrophic instability is primarily a baroclinic instability, with $P m P e>0$ energy conversion and a secondary horizontal-shear $\mathrm{KmKe}_{H}>0$ conversion. During and after the event the ageostrophic modes here have their energy generation through vertical and horizontal Reynolds stress correlations with the shear of the basic flow $\left(K m K e_{V} \geqslant K m K e_{H}>0\right)$, unlike a baroclinic instability or a geostrophic one with vanishing $K m K e_{V}$. The ageostrophic amplitude (i.e. relative magnitude of the horizontally divergent part of the velocity field) is largest in the neighbourhood of the critical surface $\left(\bar{V}=c_{p}\right)$. This location is also where the eigenmodes and basic flow most strongly interact, with a fluctuation potential vorticity flux pattern that acts to reduce the potential vorticity isopycnal gradient of the basic flow in the neighbourhood of its zero surface. This last property is most clearly seen by decomposing the raw PV flux into skew and residual components and by evaluating the diffusivity from the residual component. The residual flux is then always down-gradient. This result supports diffusive closures for the PV flux even in flows with unbalanced components.

Because of the combination of the near-degeneracy transition and the importance of $\mathrm{KmKe}_{V}>0$ beyond it, the unstable ageostrophic modes in this more generic interior shear flow $\bar{V}(x, z)$ are dissimilar from many of the previous ageostrophic instability examples in simpler flows $(\S 1)$. More commonly shared characteristics are the importance of $A-S \approx 0$ and the relatively smaller spatial scales (i.e. larger $l$ and sharper critical surface structure) in the ageostrophic modes compared to the primary geostrophic modes. Contrary to the ageostrophic baroclinic instability modes in an Eady flow, no inertia-critical surface is evident in the spatial structure of our ageostrophic instability examples, but ordinary critical surfaces do have a clear imprint. Because our computational ability to obtain eigenmodes with small scales and small growth rates is limited, we do not yet have an overview of all possible ageostrophic modes for this basic flow; rather our results provide a few examples from an as yet unknown population.

Thus, ageostrophic shear instability of balanced flows in the interior provides a viable transition route from geostrophic to ageostrophic dynamics in the regime where balance-equation evolution is not fully integrable, which may lead to forward energy cascade toward micro-scale dissipation. Much still remains to be learned about when this occurs and how significant a role it plays in the energy cycle of the ocean.

\section{Acknowledgements}

This research is supported by the National Science Foundation (OCE-1049134) and Office of Naval Research (N00014-10-1-0484).

\section{REFERENCES}

Arakawa, A. \& Moorthi, S. 1988 Baroclinic instability in vertically discrete systems. J. Atmos. Sci. 45, 1688-1707.

ARNOLDI, W. E. 1951 The principle of minimized iterations in the solution of the matrix eigenvalue problem. Q. Appl. Maths 9, 17-29.

BARTH, J. A. 1994 Short-wavelength instabilities on coastal jets and fronts. J. Geophys. Res. 99, 16095-16116. 
Boccaletti, G., Ferrari, R. \& Fox-Kemper, B. 2007 Mixed layer instabilities and restratification. J. Phys. Oceanogr. 37, 2228-2250.

Bretherton, F. P. 1966 Critical layer instability in baroclinic flows. Q. J. R. Meteorol. Soc. 92, $325-334$.

Capet, X., McWilliams, J. C., Molemaker, M. J. \& Shchepetkin, A. F. 2008 Mesoscale to submesoscale transition in the California current system. Part II. Frontal processes. J. Phys. Oceanogr. 38, 44-64.

Cerovečki, I., Plumb, R. A. \& Heres, W. 2009 Eddy transport and mixing in a wind- and buoyancy-driven jet on the sphere. J. Phys. Oceanogr. 39, 1133-1149.

Charney, J. G. 1971 Geostrophic turbulence. J. Atmos. Sci. 28, 1087-1094.

Charney, J. G. \& Phillips, N. A. 1953 Numerical integration of the quasi-geostrophic equations for barotropic and simple baroclinic flows. J. Meteorol. 10, 71-99.

Charney, J. G. \& Stern, M. E. 1962 On the stability of internal baroclinic jets in a rotating atmosphere. J. Atmos. Sci. 19, 159-172.

Craik, A. D. D. 1988 Wave Interactions and Fluid Flows. Cambridge University Press.

Dritschel, D. G. \& VANNESTE, J. 2006 Instability of a shallow-water potential-vorticity front. J. Fluid Mech. 561, 237-254.

Dritschel, D. G. \& VIÚDEZ, Á. 2003 A balanced approach to modelling rotating stably stratified geophysical flows. J. Fluid Mech. 488, 123-150.

Eliassen, A. 1983 The Charney-Stern theorem on barotropic-baroclinic instability. Pure Appl. Geophys. 121, 563-572.

GILL, A. E. 1982 Atmosphere-Ocean Dynamics. Elsevier.

Griffiths, R. W., Killworth, P. D. \& Stern, M. E. 1982 Ageostrophic instability of ocean currents. J. Fluid Mech. 117, 343-377.

GRIfFiths, R. W. \& Linden, P. F. 1982 Laboratory experiments on fronts. Geophys. Astrophys. Fluid Dyn. 19, 159-187.

Gula, J., Plougonven, R. \& Zeitlin, V. 2009 Ageostrophic instabilities of fronts in a channel in a stratified rotating fluid. J. Fluid Mech. 627, 485-507.

HAYASHI, Y.-Y. \& Young, W. R. 1987 Stable and unstable shear modes of rotating parallel flows in shallow water. J. Fluid Mech. 184, 477-504.

Hoskins, B. J. 1976 Baroclinic waves and frontogenesis. Part I. Introduction and Eady waves. Q. J. R. Meteorol. Soc. 102, 103-122.

Hoskins, B. J., McIntyre, M. E. \& Robertson, A. W. 1985 On the use and significance of isentropic potential vorticity maps. Q. J. R. Meteorol. Soc. 111, 877-946.

HuA, B. L., Moore, D. W. \& LE GentiL, S. 1997 Inertial nonlinear equilibration of equatorial flows. J. Fluid Mech. 331, 345-371.

Killworth, P. D., Paldor, N. \& Stern, M. E. 1984 Wave propagation and growth on a surface front in a two-layer geostrophic current. J. Mar. Res. 42, 761-785.

Kuo, A., Plumb, R. A. \& Marshall, J. 2005 Transformed Eulerian-mean theory. Part II. Potential vorticity homogenization and the equilibrium of a wind- and buoyancy-driven zonal flow. J. Phys. Oceanogr. 35, 175-187.

Lehoucq, R. B. \& Sorensen, D. C. 1996 Deflation techniques for an implicitly restarted Arnoldi iteration. SIAM J. Matrix Anal. Applics. 17, 789-821.

Lindzen, R. S., FARREll, B. \& TUNG, K.-K. 1980 The concept of wave overreflection and its application to baroclinic instability. J. Atmos. Sci. 37, 44-63.

MCInTyRe, M. E. 1970 Diffusive destabilisation of the baroclinic circular vortex. Geophys. Fluid Dyn. 1, 19-57.

MCWilliams, J. C. 2008 Fluid dynamics at the margin of rotational control. Environ. Fluid Mech. 8, 441-449.

McWilliams, J. C., Molemaker, M. J. \& Yavneh, I. 2004 Ageostrophic, anticyclonic instability of a geostrophic, barotropic boundary current. Phys. Fluids 16, 3720-3725.

MCWilliams, J. C. \& YAVNEH, I. 1998 Fluctuation growth and instability associated with a singularity of the balance equations. Phys. Fluids 10, 2587-2596.

McWilliams, J. C., Yavneh, I., Cullen, M. J. P. \& Gent, P. R. 1998 The breakdown of large-scale flows in rotating, stratified fluids. Phys. Fluids 10, 3178-3184. 
Ménesguen, C., Hua, B. L., Fruman, M. D. \& Schopp, R. 2009 Intermittent layering in the Atlantic equatorial deep jets. J. Mar. Res. 67.

Molemaker, M. J., McWilliams, J. C. \& YaVneh, I. 2001 Instability and equilibration of centrifugally stable stratified Taylor-Couette flow. Phys. Rev. Lett. 86, 5270-5273.

Molemaker, M. J., McWilliams, J. C. \& Yavneh, I. 2005 Baroclinic instability and loss of balance. J. Phys. Oceanogr. 35, 1505-1517.

Moore, G. W. K. \& Peltier, W. R. 1987 Cyclogenesis in frontal zones. J. Atmos. Sci. 44, 384-409.

Moore, G. W. K. \& Peltier, W. R. 1990 Nonseparable baroclinic instability. Part II. Primitive-equations dynamics. J. Atmos. Sci. 47, 1223-1242.

NAKAMURA, N. 1988 Scale selection of baroclinic instability - effects of stratification and nongeostrophy. J. Atmos. Sci. 45, 3253-3268.

Pennel, R., Stegner, A. \& Béranger, K. 2012 Shelf impact on buoyant coastal current instabilities. J. Phys. Oceanogr. 42, 39-61.

Plougonven, R., Muraki, D. J. \& Snyder, C. 2005 A baroclinic instability that couples balanced motions and gravity waves. J. Atmos. Sci. 62, 1545-1559.

Plumb, R. A. \& FerRari, R. 2005 Transformed eulerian-mean theory. Part I. Nonquasigeostrophic theory for eddies on a zonal-mean flow. J. Phys. Oceanogr. 35, 165-174.

SAKAI, S. 1989 Rossby-Kelvin instability: a new type of ageostrophic instability caused by a resonance between Rossby waves and gravity waves. J. Fluid Mech. 202, 149-176.

Salwen, H., Cotton, F. W. \& Grosch, C. E. 1980 Linear stability of Poiseuille flow in a circular pipe. J. Fluid Mech. 98, 273-284.

SAlwen, H. \& Grosch, C. E. 1972 The stability of Poiseuille flow in a pipe of circular cross-section. J. Fluid Mech. 54, 93-112.

SNYDER, C. 1995 Stability of steady fronts with uniform potential vorticity. J. Atmos. Sci. 52, 724-736.

Stone, P. H. 1970 On non-geostrophic baroclinic stability. Part II. J. Atmos. Sci. 27, 721-726.

TAYLOR, J. R. \& FERRARI, R. 2009 On the equilibration of a symmetrically unstable front via a secondary shear instability. J. Fluid Mech. 622, 103-113.

Thomas, L. N. \& TAYlor, J. R. 2010 Reduction of the usable wind-work on the general circulation by forced symmetric instability. Geophys. Res. Lett. 37, L18606.

VANneste, J. \& YAVNeH, I. 2007 Unbalanced instabilities of rapidly rotating stratified shear flows. J. Fluid Mech. 584, 373-396.

WANG, P., MCWilliams, J. C. \& KiZneR, Z. 2012 Ageostrophic instability in rotating shallow water. J. Fluid Mech. doi:10.1017/jfm.2012.422.

YAmaZaki, Y. H. \& Peltier, W. R. 2001 The existence of subsynoptic-scale baroclinic instability and the nonlinear evolution of shallow disturbances. J. Atmos. Sci. 58, 657-683.

Yavneh, I., McWilliams, J. C. \& Jeroen Molemaker, M. 2001 Non-axisymmetric instability of centrifugally stable stratified Taylor Couette flow. J. Fluid Mech. 448, 1-21. 